\title{
Prognostic impact of cigarette smoking on the survival of patients with established esophageal squamous cell carcinoma receiving radiotherapy: A retrospective study from southern China
}

\author{
GUO-RONG ZOU ${ }^{1 *}$, ZHEN SU$^{1 *}$, JUN-YUN LI², FANG-YUN XIE ${ }^{2}$ and QUN LI ${ }^{2}$ \\ ${ }^{1}$ Department of Oncology, Panyu Central Hospital, Cancer Institute of Panyu, Guangzhou, Guangdong 511400; \\ ${ }^{2}$ Department of Radiation Oncology, Sun Yat-sen University Cancer Center, State Key Laboratory of Oncology in South China, \\ Collaborative Innovation Center for Cancer Medicine, Guangzhou, Guangdong 510060, P.R. China
}

Received June 22, 2018; Accepted February 22, 2019

DOI: $10.3892 /$ etm.2019.7356

\begin{abstract}
Cigarette smoking is associated with the development of esophageal squamous cell carcinoma (ESCC); however, the influence of smoking on survival of patients with ESCC receiving radiotherapy, with or without chemotherapy, has remained elusive. The present study retrospectively analyzed 479 patients with ESCC from southern China who were categorized based on their smoking history (never, previous or current). To consider the cumulative effect of smoking, the number of pack years (PYs) was used as a representative variable. Associations between cigarette smoking and survival were evaluated using the Kaplan-Meier analysis and Cox proportional hazards model. Among the 497 patients, $308(64.3 \%)$ had reported a history of cigarette smoking. The 5-year overall survival for patients void of a smoking history, former smokers and current smokers was 50.9, 27.0 and 34.3\%, respectively. The adjusted hazard ratios (HRs) for previous and current smoking vs. no smoking history were 1.57
\end{abstract}

Correspondence to: Professor Fang-Yun Xie or Dr Qun Li, Department of Radiation Oncology, Sun Yat-sen University Cancer Center, State Key Laboratory of Oncology in South China, Collaborative Innovation Center for Cancer Medicine, 651 Dongfeng Road East, Guangzhou, Guangdong 510060, P.R. China

E-mail: xiefy0758@sina.com

E-mail: liqun0799@sina.com

*Contributed equally

Abbreviations: EC, esophageal cancer; ESCC, esophageal squamous cell carcinoma; EA, esophageal adenocarcinoma; OS, overall survival; HR, hazard ratio; CI, confidence interval; DFS, disease-free survival; RT, radiotherapy; UICC, Union for International Cancer Control; AJCC, American Joint Committee on Cancer; 2DRT, two-dimensional RT; 3DCRT, three-dimensional conformal RT; IMRT, intensity modulated RT; PY, pack year

Key words: esophageal squamous cell carcinoma, smoking, pack year, radiotherapy, survival
[95\% confidence interval (CI), 1.06-2.32] and 3.01 (95\% CI, 1.15-7.86), respectively. Heavy smokers with a high number of PYs had a HR for death of 1.75 (95\% CI, 1.28-2.41) compared with light smokers. In the cohort of 407 patients treated with intensity-modulated radiotherapy/three-dimensional conformal radiotherapy, similarly significant results were obtained. In conclusion, cigarette smoking is an independent and poor prognostic factor for patients with ESCC treated with radiotherapy and/or chemotherapy. It is associated with an increased risk of death, and the risk increases with the increase in PYs. This result may help to manage tobacco use among patients with ESCC. The smoking status should be taken into consideration in prospective studies on ESCC. More frequent follow-ups are recommended for those patients with ESCC with a history of smoking.

\section{Introduction}

Esophageal cancer (EC) is one of the most common cancer types of the digestive tract worldwide and remains one of the fourth leading causes of cancer-associated mortality in China (1,2). Esophageal squamous cell carcinoma (ESCC) and esophageal adenocarcinoma (EA) are the two major histological subtypes of EC. In China, ESCC accounts for $\sim 90 \%$ of all cases of EC, whereas EA is the predominant subtype in Western countries (3-5). Surgery is considered to be the standard treatment for this localized disease and is the best single-modality therapy for potentially this resectable disease (2). However, most patients with EC already have locally advanced or metastatic disease at the time of diagnosis. Radiotherapy (RT) combined with chemotherapy, with or without surgery, has become the major treatment (2).

Cigarette smoking is well known to promote the development of EC, irrespective of the pathological type (6,7). Most studies on the subject have revealed that smoking is a risk factor for the occurrence of ESCC (8-12). A review indicated that cigarette smoking induces a more malignant tumor phenotype by increasing the cell proliferation, migration and invasion, as well as angiogenesis, and by activating cellular pro-survival pathways (13). However, few studies have focused on the effect of smoking on EC patient survival outcomes. In 
a study by Wang et al (14), the patients underwent esophagectomy without any pre-operative therapy, and smoking was identified to be an independent prognostic factor for overall survival (OS) [hazard ratio $(\mathrm{HR})=2.186 ; 95 \%$ confidence interval (CI), 1.309-3.650; $\mathrm{P}=0.003$ ] and disease-free survival (DFS) $(\mathrm{HR}=2.471 ; 95 \% \mathrm{CI}, 1.467-4.163 ; \mathrm{P}=0.001)$. However, another study from Southern China indicated that smoking history only affected treatment outcomes in those ESCC patients receiving surgery plus chemotherapy, and not in those receiving surgery alone (15). Furthermore, one study from Shandong province reported a negative result, namely that neither smoking nor drinking affected the 2-year OS or DFS of ESCC patients (16). Considering these inconsistent results, the impact of smoking on the survival of ESCC remains elusive and the patients mainly received surgery in previous studies (14-16).

Smoking was reported as an independent predictor of a pathological complete response to neoadjuvant chemoradiotherapy in patients with ESCC (17). That study performed no further analysis of the impact of smoking on long-term survival. A recent study identified cigarette smoking as a significant and independent poor prognostic risk factor for OS among those patients with ESCC receiving definitive RT or concurrent chemoradiotherapy, with or without esophagectomy (18). In addition, smoking was demonstrated to have an unfavorable impact on tumor control by irradiation in animal models, by exacerbating tissue hypoxia $(19,20)$. Tumor hypoxia is well known to influence the reaction to radiation and chemotherapy $(21,22)$. In the present study, it was speculated that smoking not only induces malignant transformation of normal cells, but may also change tumor-associated genes or associated metabolic activity, thus making tumor cells more aggressive and less sensitive to RT and chemotherapy. Therefore, RT with or without chemotherapy, as the major treatment for those patients with local advanced ESCC, is probably affected by smoking to a greater extent than by surgery. Therefore, the aim of the present study was to elucidate the effect of a history of cigarette smoking on the survival of patients with ESCC receiving $\mathrm{RT}$, with or without chemotherapy.

\section{Patients and methods}

Patients. The medical records of the eligible patients, who were hospitalized at the Sun Yat-sen University Cancer Center (Guangzhou, China) between January 2007 and December 2013, were retrospectively reviewed. Patients were eligible if their biopsy specimens were histologically confirmed as ESCC and if they had no distant metastasis, were previously untreated and received RT with or without chemotherapy after diagnosis. Essential pre-treatment assessments were the review of the complete patient history, including a family history of cancer and lifestyle behavior; physical examination; hematology and biochemistry profiles; computed tomography of the neck, chest and upper abdomen; and endoscopic ultrasound. Patients who had distant metastasis, received surgery or had incomplete data were excluded.

The information retrieved from the medical records included age, sex, pathological type, smoking status at diagnosis (never/current/former smoker), number of cigarettes smoked per day, number of years of smoking and alcohol drinking status at diagnosis (yes vs. no). The patients' smoking status was defined as follows: Never smokers, patients who had never smoked prior to treatment; current smokers, patients who smoked prior to treatment or had stopped for $<1$ year; former smokers, patients who had stopped smoking for at least 1 year prior to treatment. The tumor locations included cervical, upper third of thoracic esophagus, middle third of thoracic esophagus and lower third of thoracic esophagus. All patients were re-staged according to the sixth edition of the Union for International Cancer Control (UICC)/American Joint Committee on Cancer (AJCC) staging system for ESCC (23).

Treatment. The treatment strategy for the patients with ESCC was discussed by a multidisciplinary team, which included surgeons, a physician, a radiation specialist, a radiologist and a pathologist. The final treatment choice was made according to the National Comprehensive Cancer Network (NCCN) guidelines and the overall condition of the patient, which included the physical performance and the economic status (2). All of the patients included received RT. The radiation techniques and dose prescriptions were in accordance with those described previously $(24,25)$. The chemotherapy consisted of fluoropyrimidine- or taxane-based regimens [cisplatin combined with 5-fluorouracil (5-FU) or cisplatin combined with docetaxel] every 3 weeks or weekly $(26,27)$. A total of 423 out of 479 $(88.3 \%)$ patients received RT plus chemotherapy. Among the patients who received chemotherapy, 110 received chemotherapy containing 5-FU, while 323 received chemotherapy containing docetaxel.

Follow-up. Patients were followed up at regular intervals after completing their treatment. The specific follow-up intervals were one month after completion of treatment, then every 2 months during the first 6 months, every 3 months for the next 6 months, every 4 months during the second year and every 6 months thereafter.

Study endpoints. The endpoint of the present study was the OS, defined as the time from treatment to death resulting from any cause. First, the association between survival and the smoking status at diagnosis (never smokers, former smokers and current smokers) was assessed. Second, the cumulative effects of smoking in terms of pack-years (PYs) were assessed. The PYs were calculated by multiplying the number of packs of cigarettes smoked per day by the number of years the patient had smoked.

Statistical analysis. Survivals rates were estimated using the Kaplan-Meier method and compared between subgroups using the log-rank test. Univariate analyses were performed to determine variables associated with OS. Multivariate analyses were performed using the Cox proportional hazards model. Comparisons of demographic, clinical and pathological variables between subgroups were performed using $\chi^{2}$ statistics, Fisher's exact test or the Kruskal-Wallis test. Continuous variables were assessed using restricted cubic splines (RCS) nested with Cox models using the RCS macro of the SAS software 9.1 (SAS Institute, Cary, NC, USA) and the cutoff scores of the continuous variables were subsequently selected based on receiver operating characteristic curve analyses. A 
two-sided $\mathrm{P}<0.05$ was considered to indicate statistical significance. Statistical analyses were performed using SPSS 22.0 (IBM Corp., Armonk, NY, USA) and SAS software 9.1.

\section{Results}

Patient characteristics, treatment and outcomes. A total of 479 patients with ESCC were included. The clinical stage distribution according to the sixth edition of the UICC/AJCC staging system for the 479 patients was as follows: Stage II, $\mathrm{n}=75$ (15.6\%); stage III, $\mathrm{n}=227(47.4 \%)$; and stage IV, $\mathrm{n}=177$ (37.0\%). Overall, 56/479 patients $(11.7 \%)$ were treated with RT alone and $423 / 479(88.3 \%)$ received RT plus chemotherapy. Of these 423 patients, $336(70.1 \%)$ received concurrent chemotherapy, $52(10.9 \%)$ received a combination of induction and concurrent chemotherapy, and 35 patients $(7.3 \%)$ received a combination of concurrent and adjuvant chemotherapy. With respect to RT, 72 patients $(15.0 \%)$ were treated with two-dimensional RT (2DRT), 298 patients (62.2\%) with three-dimensional conformal RT (3DCRT) and 109 patients (22.8\%) with intensity-modulated RT (IMRT).

Within a median follow-up duration of 27.89 months (range, 0.8-116.3 months), 286 patients died. The 1-, 2-, 3- and 5-year survival rates were $73.3,56.0,47.4$ and $39.5 \%$, respectively.

Patient characteristics. The percentage of never smokers, former smokers and current smokers in the entire cohort was $35.7 \%$ (171/479), 9.0\% (43/479) and 55.3\% (265/479), respectively. When the entire population was stratified by the smoking status, no significant differences were identified in terms of the $\mathrm{T}$-stage, $\mathrm{N}$-stage, $\mathrm{M}$-stage, clinical stage and RT techniques between the different groups. However, significant differences were observed in terms of age, sex, drinking status, tumor grade, tumor location and chemotherapy approach. Male patients were more frequent among the former and current smokers (Table I).

Kaplan-Meier analysis of the impact of smoking on survival. Former and current smokers had a poorer OS than never smokers in the entire population (Fig. 1A). The 5-year OS was $27.0 \%$ for former smokers vs. $34.3 \%$ for current smokers $(\mathrm{P}<0.001)$, and vs. $50.9 \%$ for never smokers $(\mathrm{P}<0.001)$. No significant difference in OS was identified between the former and current smokers $(\mathrm{P}=0.129)$. The small sample and no significant difference in survival $(\mathrm{P}=0.129)$ prompted us to combine the former and current smokers into a single group, whose 5-year survival rate was $32.3 \%$, which was significantly poorer than that of the never smokers $(\mathrm{P}<0.001$; Fig. 1B).

The cumulative effect of smoking also had a significant effect on the survival of patients with ESCC. In patients with a history of smoking, 47.5 PYs was identified as the cutoff value for heavy and light smokers associated with OS. Heavy smokers had a poorer 5-year OS of $16.3 \%$ compared with that of light smokers, with a 5-year OS of $38.4 \%$ (log-rank test, $\mathrm{P}<0.001$; Fig. 1C).

Among the patients treated with IMRT/3DCRT, current smokers or former smokers also had a poorer OS than never smokers [5-year OS, $56.4 \%$ for never smokers vs. $38.4 \%$ for current smokers $(\mathrm{P}<0.001)$ and vs. $30.3 \%$ for former smokers $(\mathrm{P}<0.001)$; Fig. 1D]. The significant difference compared with the never smokers still remained when current smokers and former smokers were combined (5-year OS, 36.1 vs. 58.2\%; log-rank test, $\mathrm{P}<0.001$; Fig. 1E). No significant difference was observed between the former and current smokers $(\mathrm{P}=0.101)$. Among those patients with a smoking history, heavy smokers with $>42.5$ PYs of cigarettes had a poorer 5-year OS of $22.1 \%$ compared with light smokers, with a 5 -year OS of $43.4 \%$ $(\mathrm{P}=0.006$; Fig. 1F).

Univariate analysis of the impact of cigarette smoking on survival. Among the patients with ESCC, univariate analyses identified drinking history (HR 1.57;95\% CI 1.25-1.98; $\mathrm{P}<0.001$ ), advanced $\mathrm{T}$ stage (HR 1.40; 95\% CI 1.17-1.67; $\mathrm{P}<0.001$ ), advanced $M$ stage (HR 1.54; 95\% CI 1.21-1.95; $\mathrm{P}<0.001$ ), advanced clinical stage (HR 1.62; 95\%CI 1.28-2.05; $\mathrm{P}<0.001$ ) and smoking history (HR 1.86; 95\% CI 1.42-2.44; $\mathrm{P}<0.001$ ) as significant risk factors for shorter OS (Table II). Female patients had a longer OS than male patients (HR 0.57; 95\% CI 0.41-0.79; $\mathrm{P}<0.001)$. Restricted to those patients with a smoking history, Higher PYs (>47.5) was a significant risk factor for shorter OS comparing to low PYs ( $\leq 47.5)$ (HR 1.70; 95\% CI 1.26-2.30; $\mathrm{P}=0.001)$. Similar results were found in those patients receiving 3DRT/IMRT.

Multivariate analysis of the impact of cigarette smoking on survival. The number of PYs had linear effects on OS in most cases, which was proven by the analysis using RCS nested within Cox modes (data not shown). In the multivariate analysis, the smoking status (former and current smokers vs. never smokers), T-stage and M-stage were identified as significant and independent prognostic factors for OS for the entire population and the patients treated with IMRT/3DCRT (Table III). PYs (heavy vs. light smokers) were had similar results (Table III).

In addition, the authors of the current study also assessed the association between smoking history and OS across strata of other potential predictors of patient outcome in the entire population (Table IV). The impact of the age, drinking status, tumor location or chemotherapy on the risk of death was not significantly affected by the smoking history. The effect of a history of smoking to increase the risk of death was restricted to male patients (adjusted $\mathrm{HR}=1.63$; 95\% CI, $1.08-2.45 ; \mathrm{P}=0.020$ ), as well as patients with a low degree of differentiation (adjusted HR=3.47; 95\% CI, 1.08-11.19; $\mathrm{P}=0.037$ ), a clinical stage of II/III (adjusted HR=1.44; 95\% CI, 1.02-2.04; $\mathrm{P}=0.039$ ) and treatment by 3DCRT/IMRT (adjusted $\mathrm{HR}=1.74 ; 95 \% \mathrm{CI}, 1.12-2.68 ; \mathrm{P}=0.013$ ). No significant impact was observed among female patients, possibly due to small sample sizes. Of note, a different result was obtained for patients with 2DRT: A smoking history had a positive impact on $\mathrm{OS}(\mathrm{HR}=0.34 ; 95 \% \mathrm{CI} 0.12-0.91 ; \mathrm{P}=0.033)$. This result is unexpected as it was hypothesized that a history of smoking would negatively impact OS; this notable result may be due to the small sample size, as only 72 patients received 2DRT, and among them, 17 were never smokers, 7 were former smokers and 48 were current smokers.

The impact of smoking on survival was then further assessed in detail (Table V). A smoking history ( $\mathrm{HR}=1.57$; 95\% CI, 1.06-2.32; $\mathrm{P}=0.025)$ and current smoking $(\mathrm{HR}=3.01$; $95 \% \mathrm{CI}, 1.15-7.86 ; \mathrm{P}=0.025)$ as opposed to a never-smoking 
Table I. Demographic and clinicopathological characteristics of patients with esophageal squamous cell carcinoma by status of smoking.

\begin{tabular}{|c|c|c|c|c|c|}
\hline Characteristics & All $(n=479)$ & Never smoker $(\mathrm{n}=171)$ & Former smoker $(n=43)$ & Current smoker $(n=265)$ & P-value \\
\hline Age (years) & & & & & 0.002 \\
\hline$<60$ & $234(48.9)$ & $79(46.2)$ & $11(25.6)$ & $144(54.3)$ & \\
\hline$\geq 60$ & $245(51.1)$ & $92(53.8)$ & $32(74.4)$ & $121(45.7)$ & \\
\hline Sex & & & & & $<0.001$ \\
\hline Male & $379(79.1)$ & $75(43.9)$ & $42(97.7)$ & $262(98.9)$ & \\
\hline Female & $100(20.9)$ & $96(56.1)$ & $1(2.3)$ & $3(1.1)$ & \\
\hline Drinking & & & & & $<0.001$ \\
\hline No & $294(61.4)$ & $156(91.2)$ & $22(51.2)$ & $116(43.8)$ & \\
\hline Yes & $185(38.6)$ & $15(8.8)$ & $21(48.8)$ & $149(56.2)$ & \\
\hline Tumor grade & & & & & 0.020 \\
\hline High & $124(25.9)$ & $59(34.5)$ & $11(25.6)$ & $54(20.4)$ & \\
\hline Intermediate & $278(58.0)$ & $86(50.3)$ & $27(62.8)$ & $165(62.3)$ & \\
\hline Low & $77(16.1)$ & $26(15.2)$ & $5(11.6)$ & $46(17.3)$ & \\
\hline Tumor location & & & & & 0.040 \\
\hline Cervical & $63(13.2)$ & $27(15.8)$ & $4(9.3)$ & $32(12.1)$ & \\
\hline Upper $^{\mathrm{a}}$ & $140(29.2)$ & $55(32.2)$ & $12(27.9)$ & $73(27.5)$ & \\
\hline Middle $^{\mathrm{b}}$ & $242(50.5)$ & $80(46.7)$ & $18(41.9)$ & $144(54.4)$ & \\
\hline Lower & $34(7.1)$ & $9(5.3)$ & $9(20.9)$ & $16(6.0)$ & \\
\hline T-stage & & & & & 0.768 \\
\hline $\mathrm{T} 2$ & $82(17.1)$ & $31(18.1)$ & $6(14.0)$ & $45(17.0)$ & \\
\hline $\mathrm{T} 3$ & $263(54.9)$ & $95(55.6)$ & $27(62.8)$ & $141(53.2)$ & \\
\hline $\mathrm{T} 4$ & $134(28.0)$ & $45(26.3)$ & $10(23.2)$ & 79 (29.8) & \\
\hline N-stage & & & & & 0.162 \\
\hline N0 & $53(11.1)$ & $25(14.6)$ & $3(7.0)$ & $25(9.4)$ & \\
\hline N1 & 426 (88.9) & $146(85.4)$ & $40(93.0)$ & 240 (90.6) & \\
\hline M-stage & & & & & 0.797 \\
\hline M0 & 303 (63.3) & 109 (63.7) & $29(67.4)$ & $165(62.3)$ & \\
\hline M1a & $176(36.7)$ & $62(36.3)$ & $14(32.6)$ & $100(37.7)$ & \\
\hline Clinical stage & & & & & 0.629 \\
\hline II & 75 (15.6) & 32 (18.7) & $7(16.3)$ & 36 (13.6) & \\
\hline III & $227(47.4)$ & $79(46.2)$ & $22(51.2)$ & $126(47.5)$ & \\
\hline IV & $177(37.0)$ & $60(35.1)$ & $14(32.5)$ & 103 (38.9) & \\
\hline Treatment & & & & & 0.007 \\
\hline RT alone & $56(11.7)$ & $27(15.8)$ & $11(25.6)$ & $18(6.8)$ & \\
\hline CCRT & $336(70.1)$ & $114(66.7)$ & $27(62.8)$ & 195 (73.6) & \\
\hline IC+CCRT & $52(10.9)$ & $19(11.1)$ & $3(7.0)$ & $30(11.3)$ & \\
\hline $\mathrm{CCRT}+\mathrm{AC}$ & $35(7.3)$ & $11(6.4)$ & $2(4.6)$ & $22(8.3)$ & \\
\hline RT technique & & & & & 0.151 \\
\hline 2D RT & $72(15.0)$ & 17 (9.9) & 7 (16.3) & $48(18.1)$ & \\
\hline 3DCRT & $298(62.2)$ & $108(63.2)$ & $27(62.8)$ & $163(61.5)$ & \\
\hline IMRT & $109(22.8)$ & 46 (26.9) & $9(20.9)$ & $54(20.4)$ & \\
\hline
\end{tabular}

Values are expressed as n (\%). ${ }^{\mathrm{a}}$ Upper, ${ }^{\mathrm{b}}$ middle and clower third of thoracic esophagus based on the UICC stage system. CCRT, concurrent chemoradiotherapy; IC, induction chemotherapy; AC, adjuvant chemotherapy; RT, radiation therapy; 2DRT, two-dimensional RT; 3DCRT, three-dimensional conformal RT; IMRT, intensity-modulated RT.

status, was associated with a higher of risk of death. The risk of death for heavy smokers was higher than that for light smokers, with an HR of 1.75 (95\% CI, 1.28-2.41; P<0.001). When the PYs were evaluated as a continuous variable, the 

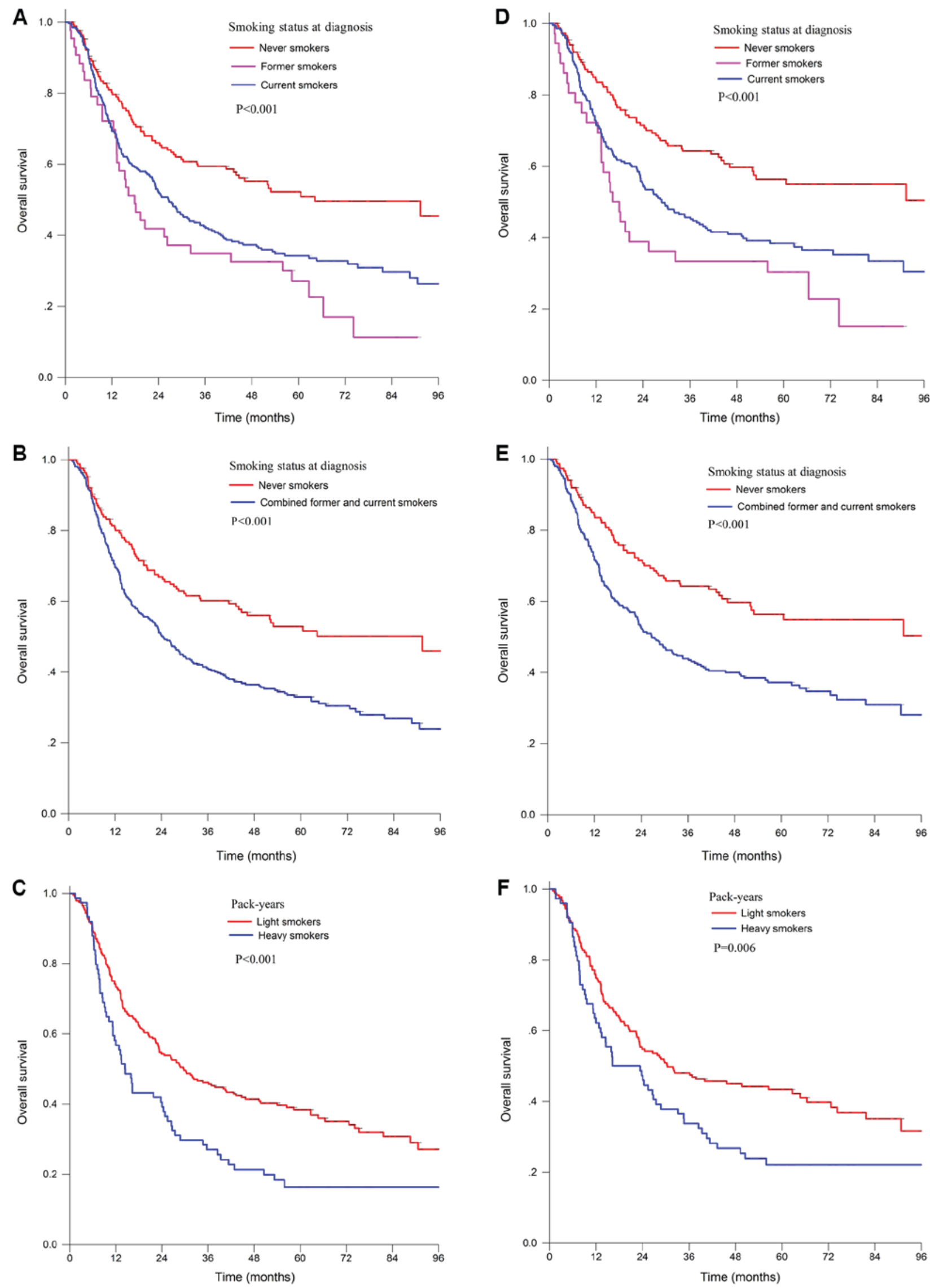

Figure 1. Kaplan-Meier curves for overall survival (A-C) in the entire study population and (D-F) the cohort subjected to IMRT or 3DRT according to smoking status at diagnosis. Patients were stratified according to (A and D) the smoking status (never, former and current), (B and E) the smoking history (no and yes) and (C and F) PYs (light and heavy). A former smoker was defined as an individual who had not smoked for 12 months or more prior to treatment A PY was defined as the equivalent of smoking one pack of cigarettes per day for 1 year. The cutoff value was 47.5 pack-years in the entire study population and 42.5 pack-years in the cohort subjected to IMRT/3DRT. PY, pack year. IMRT, intensity-modulated radiation therapy; 3DRT, three-dimensional conformal radiation therapy. 
Table II. Univariate analysis in patients with esophageal squamous cell carcinoma.

\begin{tabular}{|c|c|c|c|c|}
\hline \multirow[b]{2}{*}{ Variable } & \multicolumn{2}{|c|}{ Entire population } & \multicolumn{2}{|c|}{ IMRT/3DRT cohort } \\
\hline & $\mathrm{HR}(95 \% \mathrm{CI})$ & P-value & $\operatorname{HR}(95 \% \mathrm{CI})$ & P-value \\
\hline Age ( $\geq 60$ vs. $<60$ years) & $1.12(0.88-1.41)$ & 0.360 & $1.21(0.93-1.57)$ & 0.165 \\
\hline Sex (female vs. male) & $0.57(0.41-0.79)$ & 0.001 & $0.49(0.34-0.72)$ & $<0.001$ \\
\hline Drinking (yes vs. no) & $1.57(1.25-1.98)$ & $<0.001$ & $1.56(1.20-2.03)$ & 0.001 \\
\hline Tumor grade (high/intermediate vs. low) & $1.02(0.75-1.39)$ & 0.895 & $0.96(0.67-1.39)$ & 0.847 \\
\hline Tumor location (cervical/upper vs. middle/lower) & $1.16(0.92-1.47)$ & 0.216 & $1.15(0.88-1.51)$ & 0.289 \\
\hline T-stage (T4 vs. T3 /T2) & $1.40(1.17-1.67)$ & $<0.001$ & $1.31(1.07-1.59)$ & 0.009 \\
\hline N-stage (N1 vs. N0) & $1.48(0.98-2.24)$ & 0.060 & $2.10(1.22-3.61)$ & 0.008 \\
\hline M-stage (M1a vs. M0) & $1.54(1.21-1.95)$ & $<0.001$ & $1.55(1.18-2.02)$ & 0.001 \\
\hline Clinical stage (IV vs. II/III) & $1.62(1.28-2.05)$ & $<0.001$ & $1.62(1.24-2.12)$ & $<0.001$ \\
\hline Chemotherapy (yes vs. no) & $0.64(0.45-0.89)$ & 0.009 & $0.69(0.47-1.02)$ & 0.065 \\
\hline RT technology (IMRT/3DRT vs. 2DRT) & $0.46(0.35-0.62)$ & $<0.001$ & - & - \\
\hline Smoking history & $1.86(1.42-2.44)$ & $<0.001$ & $2.07(1.53-2.82)$ & $<0.001$ \\
\hline PYs & $1.70(1.26-2.30)^{\mathrm{a}}$ & 0.001 & $1.56(1.13-2.16)^{\mathrm{b}}$ & 0.007 \\
\hline
\end{tabular}

${ }^{\mathrm{a}} \leq \mathrm{vs} .>47.5 ;{ }^{\mathrm{b}} \leq \mathrm{vs} .>42.5$. HR, hazard ratio; CI, confidence interval; RT, radiation therapy; 2DRT, two-dimensional RT; 3DCRT, three-dimensional conformal RT; IMRT, intensity-modulated RT.

HR for death increased by $1 \%$ per pack-year $(\mathrm{HR}=1.01 ; 95 \%$ CI, 1.003-1.011; $\mathrm{P}=0.004)$.

In the subgroup of patients treated with IMRT/3DCRT, the HR for death was 1.53 (95\% CI, 1.02-2.30; $\mathrm{P}=0.039)$ for former smokers and 3.00 (95\% CI, 1.14-7.86; $\mathrm{P}=0.025)$ for current smokers, compared with that for never smokers. Heavy smokers had a higher risk of death than light smokers, with an HR of 1.55 (95\% CI, 1.11-2.16; P=0.011). Similarly, when the pack-years was evaluated as a continuous variable, the HR for death increased by $1 \%$ per pack-year $(\mathrm{HR}=1.01 ; 95 \% \mathrm{CI}$, 1.004-1.012; $\mathrm{P}=0.016$ ).

\section{Discussion}

The present study on 479 patients with ESCC receiving RT with or without chemotherapy indicated that smoking was an independent prognostic factor for poor survival after adjustment for other known prognostic factors, including age, sex, drinking status, degree of differentiation, tumor location, T-stage, N-Stage, M-stage, clinical stage, chemotherapy administration and radiation technique. The risk of death was also identified to be increased depending on the PYs of cigarettes. A specific analysis of the cohort of 407 patients treated with 3DCRT/IMRT was also performed to account for the heterogeneity of RT techniques. Considering that virtually all current smoking patients were male, an analysis based on sex was also performed revealing that smoking had a significant impact on death among male patients (adjusted $\mathrm{HR}=1.63$; $95 \%$ CI, 1.08-2.45; $\mathrm{P}=0.020$ ) but in not female patients (adjusted $\mathrm{HR}=0.40 ; 95 \% \mathrm{CI}, 0.04-3.63 ; \mathrm{P}=0.400$ ), possibly due to small sample sizes. Finally, the impact of long-term smoking on OS was assessed by treating the number of PYs as a continuous variable. The resulting HRs for death increased by a small but significant value between 0.003 and 0.011 . A previous study reported a similar result, namely that smoking decreased the OS of patients with oropharyngeal cancer by $1 \%$ per PY of smoking (28). The small sample size may be one of the reasons for the unsatisfactory interval between 0.003 and 0.011 obtained in the present study.

According to the 2014 Surgeon General's Report on smoking and tobacco use, there is sufficient evidence to infer a causal association between cigarette smoking and increased all-cause mortality and cancer-specific mortality, but is not sufficient to infer a causal association between cigarette smoking and the risk of recurrence, poorer response to treatment and increased treatment-associated toxicity (29). A review discussing the known biological effects of smoking on cancer cell biology emphasized the clinical effects of continued smoking in patients with cancer treated with chemotherapy or RT (30). Smoking causes adverse outcomes in patients with cancer, leading to complications associated with cancer treatment and continued development of comorbid disease (30). The two aforementioned studies considered lung cancer, prostate cancer, head and neck cancer, breast cancer, cervical cancer, Hodgkin's disease, colon cancer and male cancer patients $(29,30)$. However, few studies focused on patients with ESCC receiving RT with or without chemotherapy. The study by Shitara et al (31) indicated that heavy cigarette smoking (cumulative smoking of $>20 \mathrm{PYs}$ ) was a poor prognostic factor in patients with ESCC who had been treated by chemoradiotherapy. However, in their analysis, non-smokers and light smokers were combined into a group of non-heavy smokers (cumulative smoking of up to $20 \mathrm{PY}$ ), which may have introduced bias and only provides limited information on the effect of smoking behavior (31). An analysis of 1,084 patients with ESCC revealed a significant association between OS and smoking history in the group treated with chemotherapy plus surgery, but not in that treated with surgery alone (15). That 


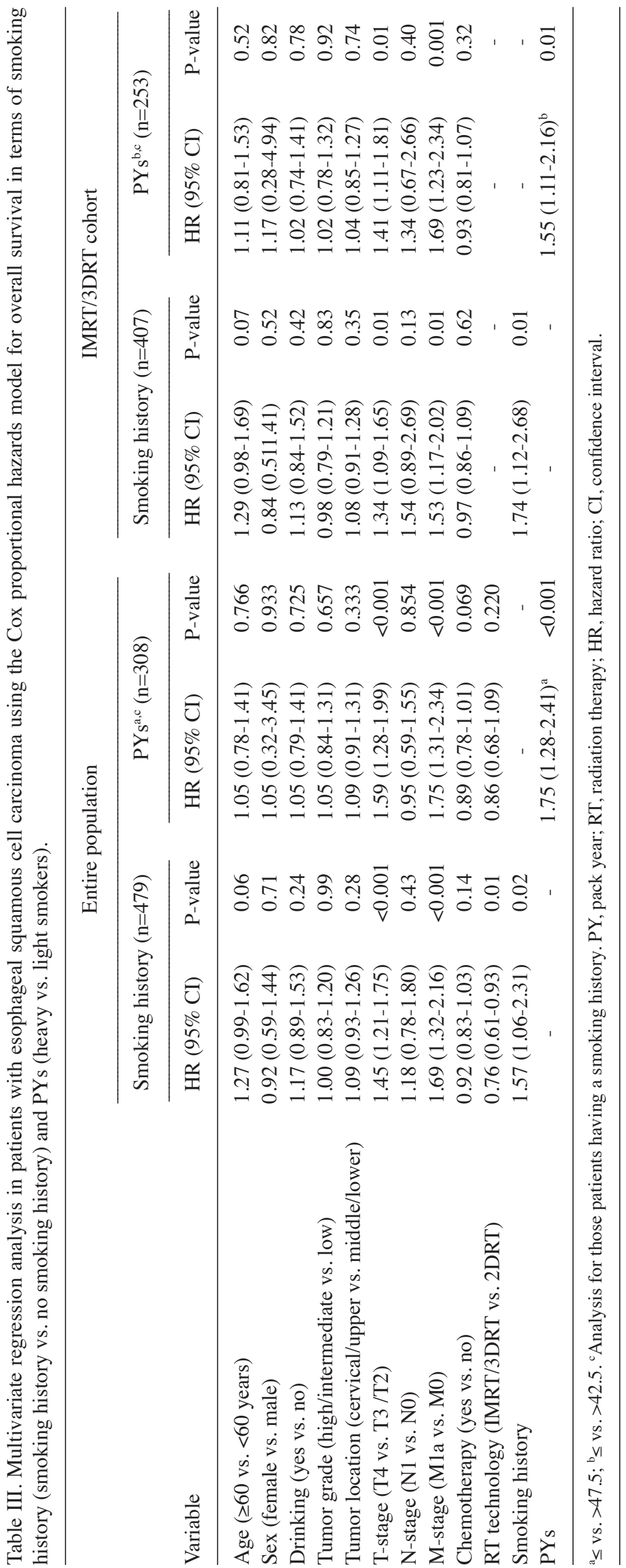


Table IV. Impact of clinicopathological characteristics on 5-year OS in the entire population and effect of the smoking history on the survival of patients with esophageal squamous cell carcinoma in subgroups by clinicopathological characteristics.

\begin{tabular}{|c|c|c|c|c|c|c|}
\hline \multirow[b]{2}{*}{ Factor } & \multirow[b]{2}{*}{$\begin{array}{l}5 \text {-year } \\
\text { OS (\%) }\end{array}$} & \multirow[b]{2}{*}{ P-value ${ }^{a}$} & \multicolumn{2}{|c|}{$\begin{array}{l}\text { No. of deaths/total } \\
\text { no. of patients } \\
\text { in the group }\end{array}$} & \multirow[b]{2}{*}{$\begin{array}{l}\text { Adjusted HR of } \\
\text { mortality }(95 \% \text { CI) }\end{array}$} & \multirow[b]{2}{*}{$\mathrm{P}$-value ${ }^{\mathrm{b}}$} \\
\hline & & & $\begin{array}{l}\text { No smoking } \\
\text { history }\end{array}$ & $\begin{array}{c}\text { Smoking } \\
\text { history }\end{array}$ & & \\
\hline Total & 39.5 & & $68 / 164$ & $218 / 315$ & $1.57(1.06-2.31)$ & 0.025 \\
\hline Age (years) & & 0.359 & & & & \\
\hline$<60$ & 41.6 & & $30 / 77$ & $107 / 157$ & $1.54(0.86-2.75)$ & 0.139 \\
\hline$\geq 60$ & 37.5 & & $38 / 87$ & $111 / 158$ & $1.57(0.91-2.70)$ & 0.102 \\
\hline Sex & & 0.001 & & & & \\
\hline Male & 35.7 & & $29 / 68$ & $215 / 311$ & $1.63(1.08-2.45)$ & 0.020 \\
\hline Female & 55.0 & & $39 / 96$ & $3 / 4$ & $0.40(0.04-3.63)$ & 0.400 \\
\hline Drinking & & $<0.001$ & & & & \\
\hline No & 47.1 & & $61 / 152$ & $91 / 142$ & $1.56(1.00-2.43)$ & 0.052 \\
\hline Yes & 28.2 & & $7 / 12$ & $127 / 173$ & $1.19(0.54-2.64)$ & 0.663 \\
\hline Degree of differentiation & & 0.895 & & & & \\
\hline High/intermediate & 39.6 & & $59 / 140$ & $179 / 261$ & $1.38(0.90-2.09)$ & 0.136 \\
\hline Low & 38.9 & & 24/96 & $53 / 97$ & $3.47(1.08-11.19)$ & 0.037 \\
\hline Tumor location & & 0.215 & & & & \\
\hline Cervical/upper ${ }^{\mathrm{c}}$ & 42.0 & & $32 / 79$ & $86 / 123$ & $1.70(0.94-3.07)$ & 0.077 \\
\hline Middle $^{\mathrm{d}} /$ lower $^{\mathrm{e}}$ & 37.7 & & $35 / 84$ & $132 / 192$ & $1.61(0.93-1.79)$ & 0.090 \\
\hline Clinical stage & & $<0.001$ & & & & \\
\hline II+III & 46.0 & & $39 / 108$ & $123 / 194$ & $1.44(1.02-2.04)$ & 0.039 \\
\hline IV & 28.1 & & $29 / 56$ & $95 / 121$ & $1.57(0.85-2.87)$ & 0.146 \\
\hline Chemotherapy & & 0.008 & & & & \\
\hline No & 21.3 & & $14 / 26$ & $25 / 30$ & $2.04(0.69-6.05)$ & 0.197 \\
\hline Yes & 41.7 & & $54 / 138$ & $193 / 285$ & $1.44(0.95-2.20)$ & 0.090 \\
\hline Radiation technique & & $<0.001$ & & & & \\
\hline 2DRT & 15.0 & & $14 / 17$ & $48 / 55$ & $0.34(0.12-0.91)$ & 0.033 \\
\hline 3DCRT/IMRT & 44.1 & & $54 / 147$ & $170 / 260$ & $1.74(1.12-2.68)$ & 0.013 \\
\hline
\end{tabular}

Adjusted HR was adjusted for age, sex, drinking status, degree of differentiation, tumor location, clinical stage, chemotherapy and radiation technique. ${ }^{\mathrm{a} P}$ for OS by each factor; ${ }^{\mathrm{b}} \mathrm{P}$ for patients with a smoking history vs. no smoking history. RT, radiation therapy; HR, hazard ratio; CI, confidence interval; 2DRT, two-dimensional RT; 3DCRT, three-dimensional conformal RT; IMRT, intensity-modulated RT; OS, overall survival. ${ }^{\mathrm{C}}$ Upper, ${ }^{\mathrm{d}}$ middle and ${ }^{\mathrm{e}}$ lower third of thoracic esophagus based on the UICC stage system.

study indicated that smoking affected the outcome of chemotherapy. In a recent study, which focused on patients receiving definitive RT or concurrent chemoradiotherapy, cigarette smoking was identified as a significant and independent poor prognostic risk factor for OS by a multivariate Cox regression analysis (18). Two groups of patients received esophagectomy after chemoradiotherapy, which may have caused bias. The study by Zhang et al (16) reported a negative result, namely that smoking was not a prognostic factor for survival of patients with ESCC who received definitive RT. However, the cohort comprised only 79 patients. In the present study, all of the patients received RT and $70.1 \%$ of patients received chemotherapy. Therefore, the present results more strongly support the view that smoking is an independent predictor of poorer survival for patients with ESCC who received RT with or without chemotherapy.

In the present study, smoking had a significant impact on the risk of death among male but not female patients, possibly due to small sample sizes. No impact of smoking on survival was observed in breast cancer patients $(32,33)$. In addition, no significant impact of smoking on OS was obtained in female patients with nasopharyngeal carcinoma (34). The reason may be that smoking in quantity and intensity is less frequent among women than among men. However, with the amount of women actively and passively smoking increasing, this association may change (35). In the present study, 100 female patients were included, of which only four of had a history of smoking. Thus, more samples of female patients are required 
Table V. Effect of smoking history on overall survival in patients with esophageal squamous cell carcinoma after adjustment for potential prognostic factors. ${ }^{a}$

Entire population

IMRT/3DCRT cohort

\begin{tabular}{|c|c|c|c|c|}
\hline \multirow[b]{2}{*}{ Variable } & & \\
\hline & HR (95\% CI) & P-value & HR $(95 \%$ CI $)$ & P-value \\
\hline \multicolumn{5}{|c|}{ Smoking status at diagnosis } \\
\hline Former vs. never & $1.57(1.06-2.32)$ & 0.025 & $1.53(1.02-2.30)$ & 0.039 \\
\hline Current vs. never & $3.01(1.15-7.86)$ & 0.025 & $3.00(1.14-7.86)$ & 0.025 \\
\hline \multicolumn{5}{|l|}{ PYs } \\
\hline Heavy vs. light & $1.75(1.28-2.41)^{\mathrm{b}}$ & $<0.001$ & $1.55(1.11-2.16)^{\mathrm{c}}$ & 0.011 \\
\hline Continuous PYs & $1.01(1.003-1.011)$ & 0.004 & $1.01(1.004-1.012)$ & 0.016 \\
\hline
\end{tabular}

${ }^{\text {a} A d j u s t e d ~ f o r ~ a g e ~(<60 ~ v s . ~}>60$ years), sex, drinking (no vs. yes), tumor location, tumor grade, T-stage, N-stage, M-stage (M0 vs. M1a) treatment and radiation technology. ${ }^{\text {bPYs: }} \leq$ vs. $>47.5$. ${ }^{\mathrm{CPY}}$ s: $\leq$ vs. $>42.5$. HR, hazard ratio; CI, confidence interval; 3DCRT, 3-dimensional conformal RT; IMRT, intensity-modulated RT; RT, radiation therapy; PYs, pack years.

to evaluate the impact of smoking on survival of patients with ESCC receiving $\mathrm{RT}$ with or without chemotherapy.

No significant difference of OS was identified between former and current smokers, and the two groups had a similarly poor survival compared with never smokers $(\mathrm{P}<0.001)$. The relatively poor survival for smokers with a higher number of PYs compared with those with a low number of PYs demonstrated the unfavorable cumulative effects of long-term, heavy smoking. The negative influence of smoking on survival was still maintained in the former smokers. Despite the cessation of smoking for $>1$ year, the possible impact of smoking on exacerbating tissue hypoxia, which induces the expression of a variety of genes associated with an aggressive malignant phenotype, and promoting chemoradioresistance and tumor progression may have already occurred and remains in these former smokers $(21,36,37)$. Furthermore, continued smoking after diagnosis may reduce the efficacy of anti-cancer treatment and increase the proportion of cancer stem-like cells, resulting in a poor outcome; in addition, increased higher rates of treatment complications and side effects, such as higher treatment-associated weight loss, lead to a poorer quality of life (38-43). A study reported that $60 \%$ of patients smoked during the week prior to surgery and $13 \%$ who were abstinent prior to surgery had resumed smoking (44). Their relapse of smoking was probably associated with a higher perceived difficulty in quitting, higher tendency toward depression, greater fears regarding cancer recurrence, a lower quitting self-efficacy and a lower perception regarding their cancer-associated risk. In addition, cessation of smoking after a cancer diagnosis was reported to significantly reduce the risk of death compared with persistent smoking (29). For the cohort of the present study, data on the smoking status during treatment or follow-up are lacking; therefore, the possibility that certain former smokers resumed smoking during treatment or follow-up cannot be excluded. Evidence-supported measures that increase chances of cessation include direct physician advice, approved pharmacotherapy, structured counseling and a follow-up plan (45). Individual behavioral counseling, a combination of pharmacological and behavioral interventions for smoking cessation, are effective in assisting smokers to quit (46).
Of note, the present study has certain limitations. First, there was an inherent bias owing to the study's retrospective design. The smoking and drinking status at diagnosis were based on the medical records, rather than standardized questionnaires at enrollment. Furthermore, in the present cohort, males accounted for the majority and few female patients had a smoking history (four smokers among 100 female patients), indicating that the present results may only apply to males. The patients included were all from Southern China, and the applicability of the present results to patients from other geographical areas remains elusive. In addition, the patients were re-staged using the sixth AJCC/UICC staging system and not the most recent staging system according to which the $\mathrm{N}$-stage is based on the number of positive lymph nodes. In the sixth AJCC/UICC staging system, N-stage was defined as with or without regional lymph node. The difference in $\mathrm{N}$-stage may change the treatment strategies. Furthermore, there was heterogeneity in the chemotherapy regimens, administration schedules and prescription doses, which ranged from 50.4 to 66.0 Gy or even higher doses ( 7 patients received $>66.0 \mathrm{~Gy}$; the maximum dose received by any one patients was $70 \mathrm{~Gy}$ ).

Finally, the treatment strategies were not entirely consistent with the latest NCCN guidelines. For instance, the T2 patients did not receive surgery due to rejection or intolerance of surgery, a group for whom surgery is the primary treatment, and some T3-T4 patients did not receive induction chemoradiotherapy followed by surgery. There were various reasons why those patients did not receive surgery. The patients with a location of the tumor in the lower esophagus, for whom surgery is the preferred treatment, only accounted for $7.1 \%$. Certain patients had comorbidities based on which they were not able to tolerate surgery. Certain patients refused surgery considering the associated complications and cost. The cohort was restricted to those subjects who received RT, with or without chemotherapy, not those who received surgery. Furthermore, induction chemotherapy followed by surgery is the standard treatment for EC according to the NCCN guidelines. The evidence that these guidelines are based on mostly comes from Western countries, in which EA is the pre-dominant subtype; however, in China, the most prevalent subtype is ESCC. 
The most common location of the tumor in Western countries is the lower esophagus, for which surgery is the preferred choice. In China, the tumor is located in the cervical and upper esophagus for most cases, for which surgery is more difficult. At our institution, the treatment strategies for the patients with ESCC were discussed by a multidisciplinary team, according to the NCCN guidelines and the status of the patients. The final treatment plan was based on the NCCN guidelines, the specific situation and the choice of each patient. Among the T2 patients in the present study, 44\% (36/82) had a tumor located in the cervical and upper esophagus, and $81.7 \%$ of cases $(67 / 82)$ were N1. To reduce the bias caused by the treatment strategy and selection, sub-group analyses were performed to evaluate the association between the smoking history and OS. In addition to an analysis of the entire population, those patients who received 3DCRT/IMRT were also assessed separately, and similarly significant results were obtained. It is important to validate the present results in a prospective study with an independent cohort.

In conclusion, the present study indicated that a smoking history at diagnosis was an independent prognostic factor for poor survival among patients with ESCC. This result may help to manage the tobacco use among patients with ESCC. The smoking status should be taken into consideration in prospective studies on ESCC. The present results require to be validated in future studies and the molecular/genetic mechanism of the effect of smoking on ESCC should be further elucidated and interpreted.

\section{Acknowledgements}

The authors greatly thank Dr. Hui He (Intensive Care Unit, Panyu Central Hospital, Cancer Institute of Panyu, Guangzhou) for his assistance in revising the figures.

\section{Funding}

No funding received.

\section{Availability of data and materials}

All data generated or analyzed during the current study are available from the corresponding author upon reasonable request.

\section{Authors' contributions}

All the authors were involved in conceiving and designing the study. GRZ, ZS and JYL collected the data. GRZ performed the statistical analysis. GRZ and ZS drafted and wrote the manuscript. FYX and QL gave advice on the study design, interpreted the results and critically revised the manuscript. All authors have read and approved the final version of the manuscript.

\section{Ethical approval and consent to participate}

This study was approved by the Ethics Committee of Sun Yat-sen University Cancer Center (Guangzhou, China) and Panyu Central Hospital (Guangzhou, China). All procedures performed in studies involving human participants were in accordance with the ethical standards of the institutional and/or national research committee and with the 1964 Helsinki Declaration and its later amendments or comparable ethical standards. Patient records were anonymized and de-identified prior to analysis. Written informed consent was obtained from all patients.

\section{Patient consent for publication}

Not applicable.

\section{Competing interests}

The authors declare that they have no competing interests.

\section{References}

1. Chen W, Zheng R, Baade PD, Zhang S, Zeng H, Bray F, Jemal A, $\mathrm{Yu}$ XQ and He J: Cancer statistics in China, 2015. CA Cancer J Clin 66: 115-132, 2016.

2. Ajani JA, D'Amico TA, Almhanna K, Bentrem DJ, Besh S, Chao J, Das P, Denlinger C, Fanta P, Fuchs CS, et al: Esophageal and esophagogastric junction cancers, version 1.2015. J Natl Compr Canc Netw 13: 194-227, 2015.

3. Chen W, Zheng R, Zhang S, Zhao P, Zeng H and Zou X: Report of cancer incidence and mortality in China, 2010. Ann Transl Med 2: 61, 2014.

4. Lin Y, Totsuka Y, He Y, Kikuchi S, Qiao Y, Ueda J, Wei W, Inoue $\mathbf{M}$ and Tanaka $\mathrm{H}$ : Epidemiology of esophageal cancer in Japan and China. J Epidemiol 23: 233-242, 2013.

5. Zhang HZ, Jin GF and Shen HB: Epidemiologic differences in esophageal cancer between Asian and Western populations. Chin J Cancer 31: 281-286, 2012.

6. Oze I, Matsuo K, Ito H, Wakai K, Nagata C, Mizoue T, Tanaka K, Tsuji I, Tamakoshi A, Sasazuki S, et al: Cigarette smoking and esophageal cancer risk: An evaluation based on a systematic review of epidemiologic evidence among the Japanese population. Jpn J Clin Oncol 42: 63-73, 2012.

7. Castellsague X, Munoz N, De Stefani E, Victora CG, Castelletto R, Rolón PA and Quintana MJ: Independent and joint effects of tobacco smoking and alcohol drinking on the risk of esophageal cancer in men and women. Int J Cancer 82: 657-664, 1999.

8. Yaegashi Y, Onoda T, Morioka S, Hashimoto T, Takeshita T, Sakata K and Tamakoshi A: Joint effects of smoking and alcohol drinking on esophageal cancer mortality in Japanese men: Findings from the Japan collaborative cohort study. Asian Pac J Cancer Prev 15: 1023-1029, 2014.

9. Lu CL, Lang HC, Luo JC, Liu CC, Lin HC, Chang FY and Lee SD: Increasing trend of the incidence of esophageal squamous cell carcinoma, but not adenocarcinoma, in Taiwan. Cancer Causes Control 21: 269-274, 2010.

10. Ishiguro S, Sasazuki S, Inoue M, Kurahashi N, Iwasaki M and Tsugane S: Effect of alcohol consumption, cigarette smoking and flushing response on esophageal cancer risk: A population-based cohort study (JPHC study). Cancer Lett 275: 240-246, 2009.

11. Jiang JM, Zeng XJ, Chen JS, Ping-zhao, Li JY, Zhang KL, Wu YP and Liu BQ: Smoking and mortality from esophageal cancer in China: A large case-control study of 19,734 male esophageal cancer deaths and 104,846 living spouse controls. Int J Cancer 119: 1427-1432, 2006.

12. Lagergren J, Bergstrom R, Lindgren A and Nyren O: The role of tobacco, snuff and alcohol use in the aetiology of cancer of the oesophagus and gastric cardia. Int J Cancer 85: 340-346, 2000.

13. Sobus SL and Warren GW: The biologic effects of cigarette smoke on cancer cells. Cancer Am Cancer Soc 120: 3617-3626, 2014

14. Wang N, Tan B, Cao F, Song Q, Wang J, Jia Y and Cheng Y: Prognostic influence of smoking on esophageal squamous cell carcinoma. Int J Clin Exp Med 8: 18867-18872, 2015.

15. Zheng Y, Cao X, Wen J, Yang H, Luo K, Liu Q, Huang Q, Chen J and Fu J: Smoking affects treatment outcome in patients with resected esophageal squamous cell carcinoma who received chemotherapy. PLoS One 10: e123246, 2015. 
16. Zhang F, Han H, Wang C, Wang J, Zhang G, Cao F and Cheng Y A retrospective study: The prognostic value of anemia, smoking and drinking in esophageal squamous cell carcinoma with primary radiotherapy. World J Surg Oncol 11: 249, 2013.

17. Huang RW, Chao YK, Wen YW, Chang HK, Tseng CK, Chan SC and Liu YH: Predictors of pathological complete response to neoadjuvant chemoradiotherapy for esophageal squamous cell carcinoma. World J Surg Oncol 12: 170, 2014.

18. Yen YC, Chang JH, Lin WC, Chiou JF, Chang YC, Chang CL, Hsu HL, Chow JM, Yuan KS, Wu ATH and Wu SY: Effectiveness of esophagectomy in patients with thoracic esophageal squamous cell carcinoma receiving definitive radiotherapy or concurrent chemoradiotherapy through intensity-modulated radiation therapy techniques. Cancer Am Cancer Soc 123: 2043-2053, 2017.

19. Jensen JA, Goodson WH, Hopf HW and Hunt TK: Cigarette smoking decreases tissue oxygen. Arch Surg 126: 1131-1134, 1991.

20. Grau C, Nordsmark M, Khalil AA, Horsman MR and Overgaard J: Effect of carbon monoxide breathing on hypoxia and radiation response in the SCCVII tumor in vivo. Int J Radiat Oncol Biol Phys 29: 449-454, 1994.

21. Cosse JP and Michiels C: Tumour hypoxia affects the responsiveness of cancer cells to chemotherapy and promotes cancer progression. Anticancer Agents Med Chem 8: 790-797, 2008

22. Nordsmark M, Bentzen SM, Rudat V, Brizel D, Lartigau E, Stadler P, Becker A, Adam M, Molls M, Dunst J, et al: Prognostic value of tumor oxygenation in 397 head and neck tumors after primary radiation therapy. An international multi-center study. Radiother Oncol 77: 18-24, 2005.

23. Fi G, Di P and Id F: AJCC cancer staging manual, 6th edition. Springer-Verlag, New York, 2002.

24. Zhang $\mathrm{P}, \mathrm{Xi}$ M, Li QQ, Hu YH, Guo X, Zhao L, Liu H, Liu SL, Luo LL, Liu Q and Liu MZ: Concurrent cisplatin and 5-fluorouracil versus concurrent cisplatin and docetaxel with radiotherapy for esophageal squamous cell carcinoma: A propensity score-matched analysis. Oncotarget 7: 44686-44694, 2016.

25. Liu H, Lu L, Zhu Q, Hao Y, Mo Y, Liu M, Hu Y, Cui N and Rong T: Cervical nodal metastases of unresectable thoracic esophagea squamous cell carcinoma: Characteristics of long-term survivors after concurrent chemoradiotherapy. Radiother Oncol 99 181-186, 2011.

26. Xi M, Zhang P, Zhang L, Yang YD, Liu SL, Li Y, Fu JH and Liu MZ: Comparing docetaxel plus cisplatin versus fluorouracil plus cisplatin in esophageal squamous cell carcinoma treated with neoadjuvant chemoradiotherapy. Jpn J Clin Oncol 47: 683-689, 2017

27. Luo LL, Xi M, Yang YD, Li QQ, Zhao L, Zhang P, Liu SL and Liu MZ: Comparative outcomes of induction chemotherapy followed by definitive chemoradiotherapy versus chemoradiotherapy alone in esophageal squamous cell carcinoma. J Cancer 8: 3441-3447, 2017.

28. Gillison ML, Zhang Q, Jordan R, Xiao W, Westra WH, Trotti A, Spencer S, Harris J, Chung CH and Ang KK: Tobacco smoking and increased risk of death and progression for patients with p16-positive and p16-negative oropharyngeal cancer. J Clin Oncol 30: 2102-2111, 2012 .

29. Warren GW, Alberg AJ, Kraft AS and Cummings KM: The 2014 surgeon General's report: 'The health consequences of smoking-50 years of progress': A paradigm shift in cancer care. Cancer 120: 1914-1916, 2014

30. Warren GW, Sobus S and Gritz ER: The biological and clinical effects of smoking by patients with cancer and strategies to implement evidence-based tobacco cessation support. Lancet Oncol 15: e568-e580, 2014
31. Shitara K, Matsuo K, Hatooka S, Ura T, Takahari D, Yokota T, Abe T, Kawai H, Tajika M, Kodaira T, et al: Heavy smoking history interacts with chemoradiotherapy for esophageal cancer prognosis: A retrospective study. Cancer Sci 101: 1001-1006, 2010.

32. Vatten LJ, Foss OP and Kvinnsland S: Overall survival of breast cancer patients in relation to preclinically determined total serum cholesterol, body mass index, height and cigarette smoking: A population-based study. Eur J Cancer 27: 641-646, 1991.

33. Terry PD and Rohan TE: Cigarette smoking and the risk of breast cancer in women: A review of the literature. Cancer Epidemiol Biomarkers Prev 11: 953-971, 2002.

34. Ouyang PY, Su Z, Mao YP, Liang XX, Liu Q, Deng W and Xie FY: Prognostic impact of cigarette smoking on the survival of patients with established nasopharyngeal carcinoma. Cancer Epidemiol Biomarkers Prev 22: 2285-2294, 2013.

35. Johnson KC, Miller AB, Collishaw NE, Palmer JR, Hammond SK, Salmon AG, Cantor KP, Miller MD, Boyd NF, Millar J and Turcotte F: Active smoking and secondhand smoke increase breast cancer risk: The report of the Canadian expert panel on tobacco smoke and breast cancer risk (2009). Tob Control 20: e2, 2011.

36. Brizel DM, Sibley GS, Prosnitz LR, Scher RL and Dewhirst MW: Tumor hypoxia adversely affects the prognosis of carcinoma of the head and neck. Int J Radiat Oncol Biol Phys 38: 285-289, 1997.

37. Semenza GL: Hypoxia-inducible factors: Mediators of cancer progression and targets for cancer therapy. Trends Pharmacol Sci 33: 207-214, 2012.

38. An Y, Kiang A, Lopez JP, Kuo SZ, Yu MA, Abhold EL, Chen JS, Wang-Rodriguez J and Ongkeko WM: Cigarette smoke promotes drug resistance and expansion of cancer stem cell-like side population. PLoS One 7: e47919, 2012.

39. Zevallos JP, Mallen MJ, Lam CY, Karam-Hage M, Blalock J, Wetter DW, Garden AS, Sturgis EM and Cinciripini PM: Complications of radiotherapy in laryngopharyngeal cancer: Effects of a prospective smoking cessation program. Cancer 115: 4636-4644, 2009.

40. Arcavi L and Benowitz NL: Cigarette smoking and infection. Arch Intern Med 164: 2206-2216, 2004.

41. Duffy SA, Terrell JE, Valenstein M, Ronis DL, Copeland LA and Connors M: Effect of smoking, alcohol, and depression on the quality of life of head and neck cancer patients. Gen Hosp Psychiatry 24: 140-147, 2002.

42. Browman GP, Wong G, Hodson I, Sathya J, Russell R, McAlpine L, Skingley P and Levine MN: Influence of cigarette smoking on the efficacy of radiation therapy in head and neck cancer. N Engl J Med 328: 159-163, 1993.

43. Gritz ER: Smoking and smoking cessation in cancer patients. $\mathrm{Br}$ J Addict 86: 549-554, 1991

44. Simmons VN, Litvin EB, Jacobsen PB, Patel RD, McCaffrey JC, Oliver JA, Sutton SK and Brandon TH: Predictors of smoking relapse in patients with thoracic cancer or head and neck cancer. Cancer 119: 1420-1427, 2013.

45. Steliga MA: Smoking cessation in clinical practice: How to get patients to stop. Semin Thorac Cardiovasc Surg 30: 87-91, 2018.

46. Lancaster T and Stead LF: Individual behavioural counselling for smoking cessation. Cochrane Database Syst Rev 3: CD001292, 2017.

This work is licensed under a Creative Commons Attribution-NonCommercial-NoDerivatives 4.0 International (CC BY-NC-ND 4.0) License. 